\title{
Structural and Functional Elucidation of IF-3 Protein of Chloroflexus aurantiacus Involved in Protein Biosynthesis: An In Silico Approach
}

\author{
Abu Saim Mohammad Saikat $\mathbb{D}^{1},{ }^{1}$ Md. Ekhlas Uddin $\mathbb{D}^{2},{ }^{2}$ Tasnim Ahmad $\mathbb{D},{ }^{3}$ \\ Shahriar Mahmud $\mathbb{D}^{3}$ Md. Abu Sayeed Imran $\mathbb{D}^{3}{ }^{3}$ Sohel Ahmed $\mathbb{D}^{4},{ }^{4}$ Salem A. Alyami $\mathbb{D}^{5}$ \\ and Mohammad Ali Moni ${ }^{6,7}$ \\ ${ }^{1}$ Department of Biochemistry and Molecular Biology, Bangabandhu Sheikh Mujibur Rahman Science and Technology University, \\ Gopalganj 8100, Bangladesh \\ ${ }^{2}$ Department of Biochemistry and Molecular Biology, Gono University, Dhaka 1344, Bangladesh \\ ${ }^{3}$ Department of Biotechnology and Genetic Engineering, Islamic University, Jhenidah-Kushtia, Bangladesh \\ ${ }^{4}$ Department of Biochemistry and Molecular Biology, Jahangirnagar University, Dhaka 1342, Bangladesh \\ ${ }^{5}$ Department of Mathematics and Statistics, Faculty of Science, Imam Mohammad Ibn Saud Islamic University (IMSIU), \\ Riyadh 13318, Saudi Arabia \\ ${ }^{6}$ School of Psychiatry, Faculty of Medicine, University of New South Wales, Sydney, NSW 2052, Australia \\ ${ }^{7}$ Healthy Ageing Theme, The Garvan Institute of Medical Research, Darlinghurst, NSW 2010, Australia
}

Correspondence should be addressed to Md. Ekhlas Uddin; dipubmbgb@gmail.com and Mohammad Ali Moni; m.moni@unsw.edu.au

Received 6 April 2021; Revised 9 June 2021; Accepted 14 June 2021; Published 2 July 2021

Academic Editor: Rita Casadio

Copyright (c) 2021 Abu Saim Mohammad Saikat et al. This is an open access article distributed under the Creative Commons Attribution License, which permits unrestricted use, distribution, and reproduction in any medium, provided the original work is properly cited.

\begin{abstract}
Chloroflexus aurantiacus is a thermophilic bacterium that produces a multitude of proteins within its genome. Bioinformatics strategies can facilitate comprehending this organism through functional and structural interpretation assessments. This study is aimed at allocating the structure and function through an in silico approach required for bacterial protein biosynthesis. This in silico viewpoint provides copious properties, including the physicochemical properties, subcellular location, three-dimensional structure, protein-protein interactions, and functional elucidation of the protein (WP_012256288.1). The STRING program is utilized for the explication of protein-protein interactions. The in silico investigation documented the protein's hydrophilic nature with predominantly alpha $(\alpha)$ helices in its secondary structure. The tertiary-structure model of the protein has been shown to exhibit reasonably high consistency based on various quality assessment methods. The functional interpretation suggested that the protein can act as a translation initiation factor, a protein required for translation and protein biosynthesis. Protein-protein interactions also demonstrated high credence that the protein interconnected with $30 \mathrm{~S}$ ribosomal subunit involved in protein synthesis. This study bioinformatically examined that the protein (WP_012256288.1) is affiliated in protein biosynthesis as a translation initiation factor IF-3 of C. aurantiacus.
\end{abstract}

\section{Introduction}

In specific, Chloroflexus aurantiacus is a Gram-negative organism possessing exceptional characteristics, such as anoxygenic, filamentous, thermophilic, phototrophic, and gliding properties [1-3]. Keeping out other phototrophic anoxygenic, Chloroflexus aurantiacus sprout effectively in environments with a moderate temperature of $50-60^{\circ} \mathrm{C}[4$, $5]$. They can mostly acclimatize in various environmental circumstances, including wetlands, river water, hot springs, and sediments containing elevated-sulfide conditions $[6,7]$. Surprisingly, the species of bacteria have specific similar 
TABle 1: Protein retrieval.

\begin{tabular}{lc}
\hline Protein individualities & Protein information \\
\hline Locus & WP_012256288 \\
Amino acid & 275 aa \\
Definition & Translation initiation factor IF-3 [Chloroflexus aurantiacus] \\
Accession & WP_012256288 \\
Version & WP_012256288.1 \\
Source & Chloroflexus aurantiacus \\
Keywords & RefSeq \\
Organism & Chloroflexus aurantiacus \\
& >WP_012256288.1 translation initiation factor IF-3 [Chloroflexus aurantiacus] \\
& MPRLSPVARRRSRAR \\
& DRFRINNRIRAREVRLID \\
FASTA sequence & ENGTQVGIVPLREALAMAEERGFLV \\
& EVAPNAVPPVCRLLDYGKFREQSKKEREARRN \\
& QKQSELKQIRLMPKTDDHDVAVKANQARRFLAG \\
& DKVKFNLRFGREMAHPEIGRQMLDQIAEQLSDI \\
& AVIEQKPLMEGRVLSMLLAPTAKVLKAAQ \\
\end{tabular}

TABle 2: Physicochemical parameters.

\begin{tabular}{ll}
\hline Parameters & Value \\
\hline $\begin{array}{l}\text { Molecular weight } \\
\text { Theoretical pI }\end{array}$ & 31444.01 \\
$\begin{array}{l}\text { Total number of negatively } \\
\text { charged residues (Asp+Glu) }\end{array}$ & $62.88,4.62^{*}$ \\
$\begin{array}{l}\text { Total number of positively } \\
\text { charged residues (Arg+Lys) }\end{array}$ & 48 \\
$\begin{array}{l}\text { Formula } \\
\text { Total number of atoms }\end{array}$ & $\mathrm{C}_{1336} \mathrm{H}_{2179} \mathrm{~N}_{417} \mathrm{O}_{444} \mathrm{~S}_{8}$ \\
& 4384 \\
& (a) 30 hours (mammalian \\
rhe estimated half-life & (b) $>20$ hours (yeast, in vivo) \\
& (c) $>10$ hours (Escherichia \\
coli, in vivo) \\
$\begin{array}{l}\text { Instability index (II) } \\
\text { Aliphatic index }\end{array}$ \\
$\begin{array}{l}\text { Grand average of hydropathicity } \\
\text { (GRAVY) }\end{array}$ & 73.38 \\
\hline
\end{tabular}

*pI calculated by the SMS v.2.0.

characteristics, particularly chimeric photosystem, with purple-photosynthetic bacteria (PPB) and green-sulfur bacteria (GSB) $[8,9]$. Placed in a certain way, the Chloroflexi are the first expanding bacteria that can generate their nutrients using photosynthesis [8]. Regarding the remarkable photosynthetic and thermophilic properties, the bacterium compelled investigators to examine multiple proteins involved with heat tolerance, formulating industrially crucial enzymes including propionyl-CoA synthase [10], maltotetraose-producing amylase [11], malonyl-CoA reductase, and so on in recent years [12]. Additionally, highlighting the genome's special features has attracted much attention by studying genome repositories.
TABLE 3: Subcellular localization assessment.

\begin{tabular}{lc}
\hline Analysis & Result \\
\hline CELLO (v.2.5) & Cytoplasmic \\
PSORTb (v.3.0.2) Cytoplasmic \\
SOSUI $_{\text {Gram }} \mathrm{N}$ & Cytoplasmic \\
PSLpred & Cytoplasmic \\
HMMTOP (v.2.0) & No transmembrane helices present \\
TMHMM (v.2.0) & No transmembrane helices present \\
\hline
\end{tabular}

Due to advances in computational biology, various platforms and methods have been built for predicting protein structure, recognizing sequence similarities performing phylogenic research, analyzing active site residue correlation, protein-ligand interaction, protein-protein interaction, gene expression screening, motif phosphorylation area recognition, and conserved domain determination [13-16]. A study using bioinformatics methods of the proteins allows one to evaluate their three-dimensional structural conformation, classify new domains, examine specific pathways to obtain a perspective of our evolutionary tree, identify additional clusters, and attach the proteins' role [17]. This accomplished knowledge can also impart effective pharmacological strategies and assistance in prospective drug design against many diseases [18-20].

The protein translation initiation factor IF-3 (WP 012256288.1) is deeply associated with protein biosynthesis in C. aurantiacus. The translation is the final phase of gene expression, which involves translating DNA into RNA and using the RNA to create amino acid chains. Translation includes four distinct stages. These phases include a pretranslational step, initial elongation, termination, and ribosome retrieval. Throughout each step, ribosomes interact with allied translation elements to relay signals essential for 


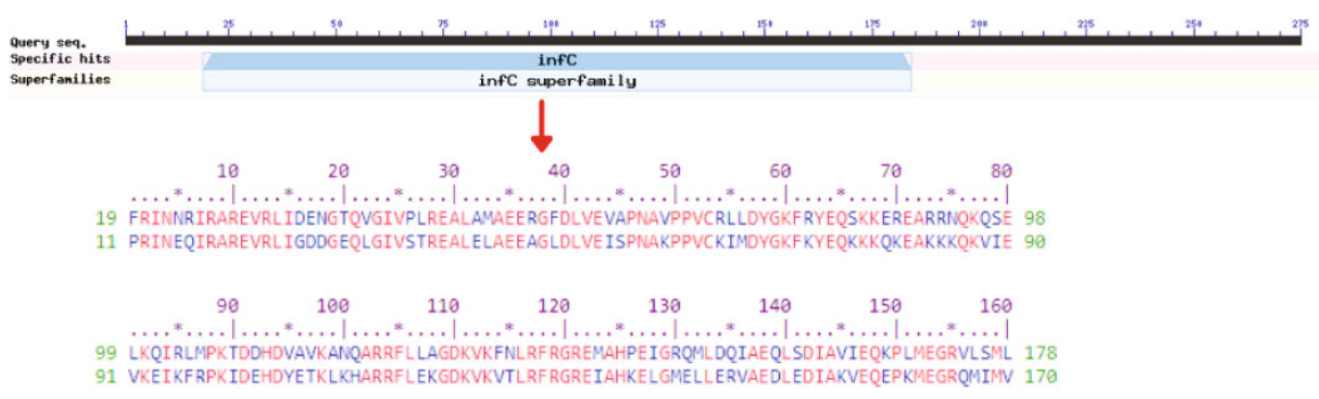

(a)

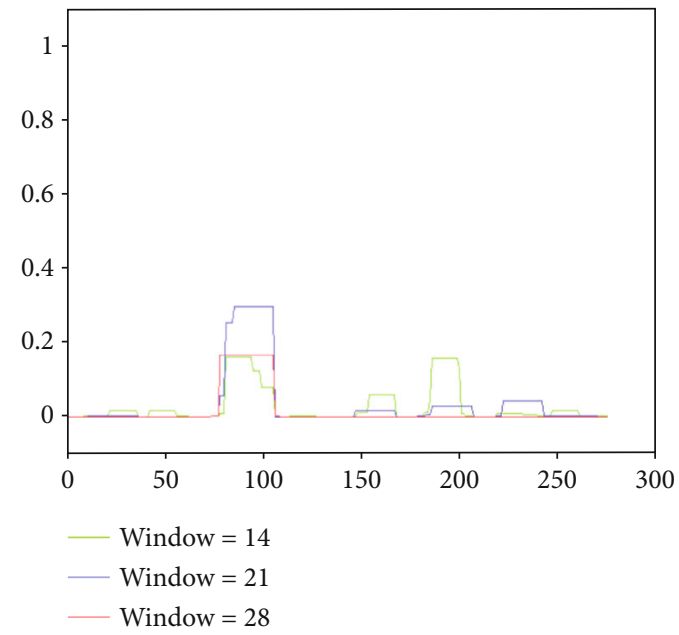

(b)

FIGURE 1: (a) Functional annotation of the protein WP_012256288.1. The graphical summary represents the conserved domains identified in the query sequence. The aligned sequences represent the conserved domains identified on the query sequence by comparing with the conserved protein domain family, infC (CDD accession no. PRK00028). The ScanProsite predicted a motif at $72-85$ (accession no. PS00938) as infC, whereas the Pfam demonstrated two, including the C-terminal and N-terminal domain at 98-181 and 2-90 positions, respectively. The SuperFamily program predicted the protein as a member of the infC superfamily. Moreover, (b) coil reveals the heptads of existing windows 14 (green color), 21 (blue color), and 28 (red color). The $x$-axis of the diagram displays the adjustment in the protein of amino acid number (beginning at the $\mathrm{N}$-terminus), whereas the $y$-axis indicates the spinning coil, while "window" corresponds to the width of the amino acid window, which is inspected concurrently.

protein formulation. It is also crucial to know that the ribosome's conformational mechanisms, translation stimuli, and ribosomal complexes perform a crucial function in directing the translation system's directionality. A key obstacle for the scientists is to grasp how the poorly combined movements of the translational elements contribute to right and rapid protein synthesis [21]. IF-3 is one of the crucial elements required to stimulate the start of protein synthesis in prokaryotes. IF-3 attaches to the $30 \mathrm{~S}$ ribosomal subunit (RS) and switches the balance between the $70 \mathrm{~S}$ ribosomes and their available subunits in a manner that enhances the supply of free subunits, thereby maximizing the abundance of novel proteins ready to be constructed [22-24].

Additionally, this assessment enables the recognition of novel biotechnological targets through an adaptive mechanism that involves functional annotation, contemporary gene annotation, and three-dimensional protein modeling.

\section{Methodology}

2.1. Protein Selection and Sequence Retrieval. The amino acid (aa) sequence of the translation initiation factor IF-3 protein present in Chloroflexus aurantiacus was retrieved from the NCBI database (https://www.ncbi.nlm.nih.gov/) in FASTA format.

2.2. Physicochemical Characterization. The physicochemical parameters of the protein (WP_012256288.1) were evaluated by the ProtParam assessment tool of ExPASy server (https:// web.expasy.org/protparam/) and the SMS v.2.0server (https://www.bioinformatics.org/sms2/).

2.3. Subcellular Location Identification. The subcellular location of the protein was documented by utilizing the CELLO v.2.5 [25, 26], PSORTb v.3.0.2 [27], SOSUI assessment tool 


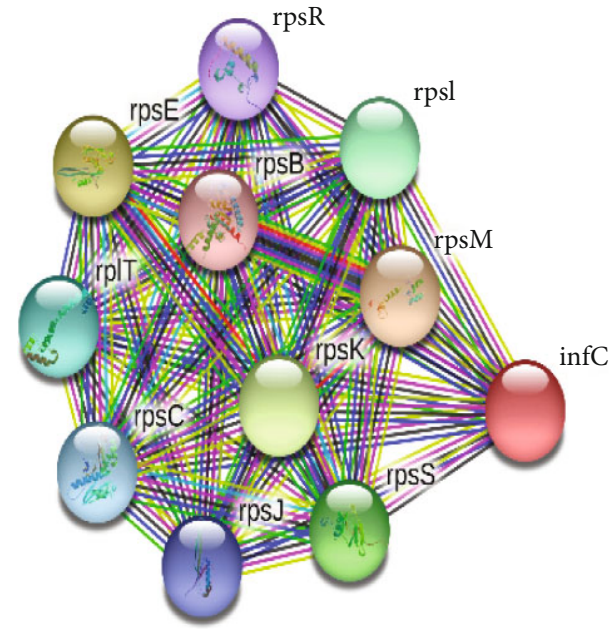

FIGURE 2: The STRING network of the protein determines the protein-protein interactions. The rpsM, rpsE, rpsK, rpsS, rpsI, rpIT, rpsC, rpsJ, rpsR, and rpsB represent the $30 \mathrm{~S}$ ribosomal protein S13, 30S ribosomal protein S5, 30S ribosomal protein S11, 30 S ribosomal protein S19, ribosomal protein S9, 50S ribosomal protein L20, 30S ribosomal protein S3, 30S ribosomal protein S10, 30 S ribosomal protein S18, and ribosomal protein, respectively. Colored nodes represent query proteins and the first shell of interactors, and white nodes describe the second shell of interactors. For node content: empty nodes designate proteins of unknown 3D structure, and filled nodes render some 3D structure as known or predicted.

[28], PSLpred server [29], HMMTOP v.2.0 [30], and TMHMM server v.2.0 (http://www.cbs.dtu.dk/services/TMHMM/).

2.4. Functional Annotation Prediction. The NCBI platform's CD search tool [31] was utilized to predict the conserved domain in the protein WP_012256288.1. Protein motif determination was performed using the GenomeNet (Motif) server [32], Pfam tool [33], and ScanProsite tool (https:// prosite.expasy.org/scanprosite/) of the ExPASy program, and the SuperFamily program [34] assigned the evolutionary relationships of the protein WP_012256288.1.

2.5. Protein-Protein Interaction. The STRING v.11.0 program [35] was used for determining the possible protein-protein (pr-pr) interactions.

2.6. Secondary Structural Assessment. The SOPMA tool [36] utilized the secondary structural elements' prediction following the default parameters (window width of 17 , number of states of 4 , and the similarity threshold of 8 ) of the protein translation initiation factor IF-3 present C. aurantiacus. The SPIPRED v.4.0 [37] and the DISOPRED v.3.0 [38] tools were utilized to predict the secondary structure and the disordered areas, respectively.

2.7. Three-Dimensional Structure Prediction and Validation. HHpred predicted the three-dimensional (tertiary) structure with Modeller [39-41]. The most suitable template (HHpred ID: 5LMN_X; PDB ID: 5LMN) was selected for designing the tertiary structure among the number of hits of 130 with the probability, $E$ value, aligned cols, and target lengths of 100 ,
TABLE 4: Secondary structural elements.

\begin{tabular}{lc}
\hline Structural elements & Values (\%) \\
\hline Alpha helix (Hh) & $121(44.00)$ \\
$3_{10}$ helix (Gg) & 0 \\
Pi helix (Ii) & 0 \\
Extended strand (Ee) & $45(16.36)$ \\
Beta bridge (Bb) & $0(0.00)$ \\
Bend region (Ss) & $0(0.00)$ \\
Beta turn (Tt) & $23(8.36)$ \\
Random coil (Cc) & $86(31.27)$ \\
Ambiguous states & 0 \\
Other states & 0 \\
\hline
\end{tabular}

$2.5 \times 10^{-37}, 168$, and 171 , respectively. The PROCHECK [42] of the SAVES v.6.0 program (https://saves.mbi.ucla .edu/) was performed to predict the Ramachandran plot and validate the predicted tertiary structure.

2.8. Active Site Determination. The CASTp v.3.0 server [43] was used to predict the active sites of the modeled protein.

\section{Results and Discussion}

3.1. Sequence Retrieval. The amino acid (aa) sequence of the protein (WP_012256288.1) of C. aurantiacus was gathered from the NCBI database. The protein contains 275 amino acids. Further information on the protein (WP 012256288.1) is mentioned in Table 1.

3.2. Physicochemical Properties. Through studying the characteristics of each of the amino acids in the protein, it can be understood how the physicochemical features of the protein are defined. The ProtParam program of the ExPASy server was utilized to define the physicochemical properties of the protein (WP_012256288.1). The protein is consist of 275 amino acids where $\operatorname{Arg}(34)$ was the most abundant amino acid followed by Ala (33), Asp (33), Glu (29), Leu (20), Val (17), Lys (14), Gln (14), Pro (13), Ile (11), Thr (10), Gly (9), Ser (9), Asn (8), Phe (8), Met (7), Tyr (3), His (2), and Cys (1). There was no amino acid residue tryptophan (Trp) in the protein. Protein half-life is characterized as the period it requires for the radio-labeled focus protein density to be decreased by 50 percent compared to the amount at the onset of the chase [44]. The protein (WP_012256288.1) C. aurantiacus has an estimated half-life of about 30 hours (mammalian reticulocytes, in vitro), $>20$ hours (yeast, in vivo), and $>10$ hours (Escherichia coli, in vivo). The calculated isoelectric point (pI), molecular weight, and the total number of atoms were $4.88\left(4.62^{*}\right)$, 31444.01 Dalton, and 4384, respectively (Table 2).

Besides, the molecular formula of the protein was $\mathrm{C}_{1336} \mathrm{H}_{2179} \mathrm{~N}_{417} \mathrm{O}_{444} \mathrm{~S}_{8}$. The $\mathrm{pI}$ value introduced the protein is negatively charged where the total number of negatively charged residues $(\mathrm{Asp}+\mathrm{Glu})$ was 62 , and the total number of positively charged residues (Arg+Lys) was 48. Other parameters, including the instability index (II), describe the proteins' stability, whereas the aliphatic index (73.89) 
10

30

40

50

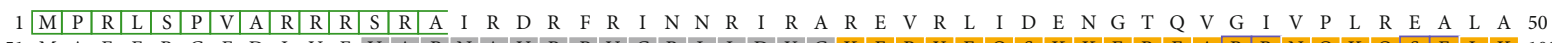

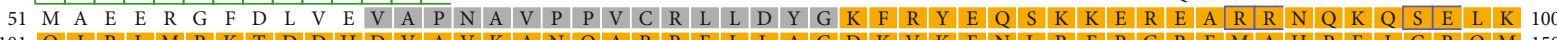

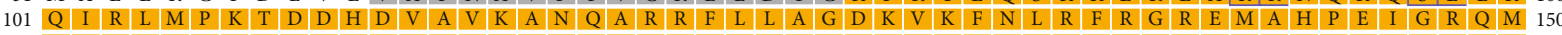

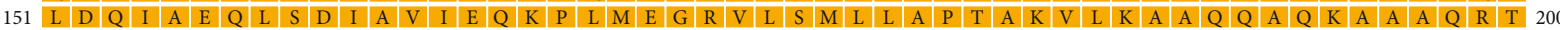

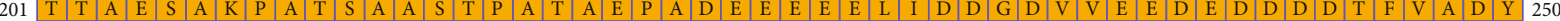
\begin{tabular}{ll|l|l|l|l|l|l|l|l|l|l|l|l|l|l|l|l|l|l|l|l|l|l|l|l|}
251 & $\mathrm{D}$ & $\mathrm{D}$ & $\mathrm{E}$ & $\mathrm{D}$ & $\mathrm{D}$ & $\mathrm{D}$ & $\mathrm{F}$ & $\mathrm{E}$ & $\mathrm{D}$ & $\mathrm{D}$ & $\mathrm{D}$ & $\mathrm{D}$ & $\mathrm{D}$ & $\mathrm{D}$ & $\mathrm{E}$ & $\mathrm{D}$ & $\mathrm{D}$ & $\mathrm{E}$ & $\mathrm{R}$ & $\mathrm{N}$ & $\mathrm{R}$ & $\mathrm{R}$ & $\mathrm{R}$ & $\mathrm{R}$ & $\mathrm{R}$ \\
\hline
\end{tabular}

10

$$
20
$$

30

40

50

Strand

Disordered, protein binding

Extracellular

Helix

Putative domain boundary

Re-entrant helix

Coil

Membrane interaction

Cytoplasmic

Disordered

Transmembrane helix

Signal peptide

(a)

Conf
Cart

Pred C C C C C C C C C C C C C C H H C C C C H H H C C C C C E E E E E C C C C C E E E E E E H H H H H H

AA M P R L P V A R R R R A I R S R R I N N R I R A R E V R L I D E N G T Q V G I V P L R A L A

10

30

40

50

Conf

H H H H H H H H H H H H C C C C E E E

AA MA E E R G F D L V E V A P N A V P P V C R L L D Y GK F R Y E Q S K K E R E A R R Q K Q S E L K

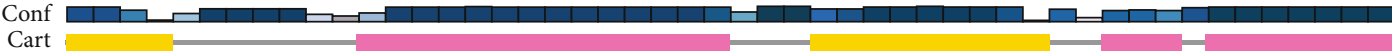

Pred E E E C C C C C C C H H H H H H H H H H H C C C E E E E E E E E C C H H H H H H H H H

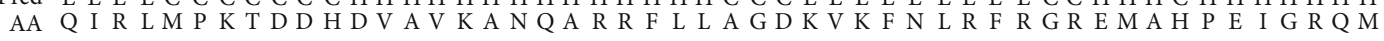

110

120

130

140

150

Conf
Cart

Pred $\mathrm{HHH} H \mathrm{HH} H \mathrm{HHC} C \mathrm{C} C \mathrm{C} C \mathrm{C} C \mathrm{C} C \mathrm{C} C \mathrm{C}$ E E E E E E C C C H H H H H H H H H H H H H

AA L D Q I A Q L D A V I Q P C

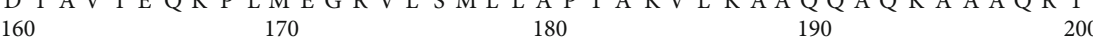

Conf

Pred H H H H C C C C C C C C C C C C C C C C C C H H H H H H C C C C C C C C C C C C C C C C C C C C C

AA $T$ T A E S A K P A T S A A S T P A T A E P A D E E E E E L I D D G D V V E E D E D D D D T F V A D Y

210

220

230

240

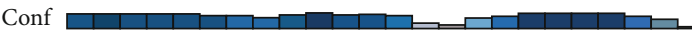

Pred C C C C C C C C C C C C C C H H H H H H H H H C C

AA D D E D D D F E D D D D D E D D E R R R R R

260

270

280

290

300

Conf: - + Confidence of prediction

Cart: 3-State assignment cartoon

Pred: 3-State prediction

AA: Target sequence

$$
\begin{aligned}
& \text { Strand } \\
& \text { Helix } \\
& \text { Coil }
\end{aligned}
$$

(b)

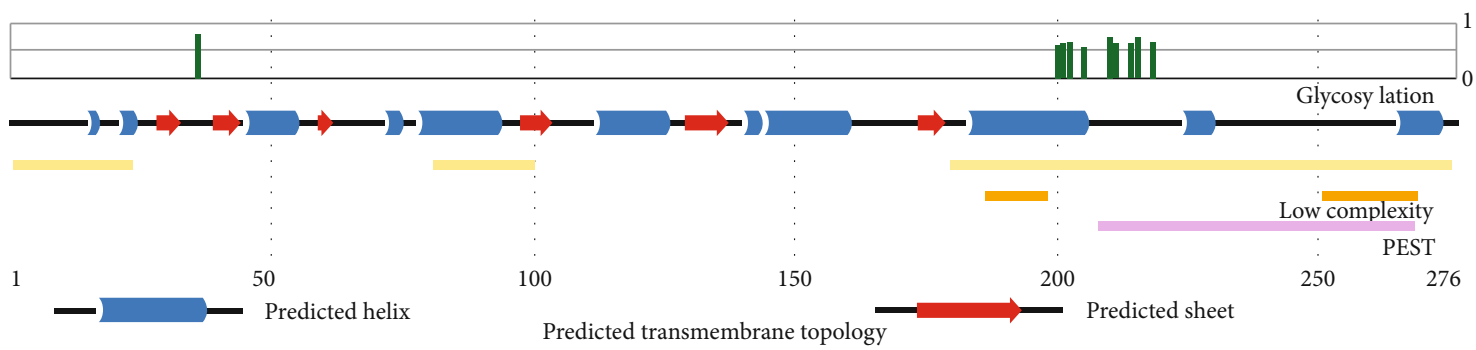

(c)

FIGURE 3: The secondary structural assessment: (a) sequence plot, (b) the predicted secondary structure, and (c) predicted transmembrane topology (position-dependent feature predictions are mapped onto the sequence schematic phenomena; the line height of the phosphorylation and glycosylation features reflects the confidence of the residue prediction). 

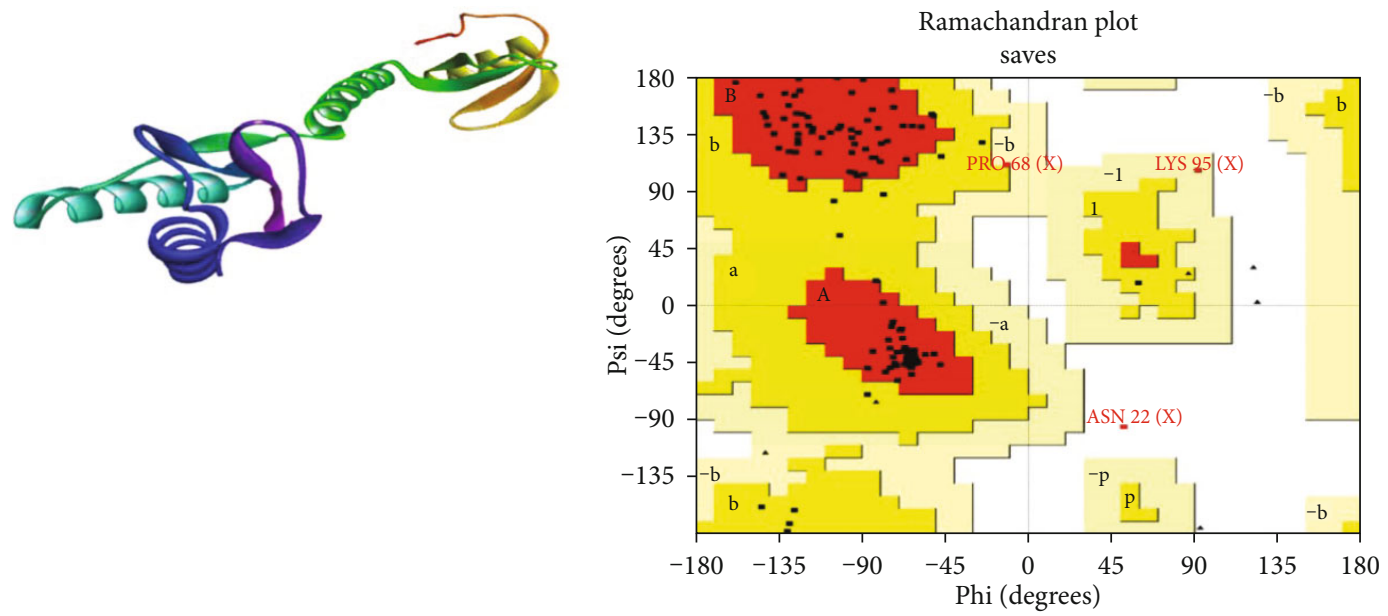

(a)

(b)

Figure 4: Tertiary structure prediction. (a) Predicted tertiary structure by HHpred tool employing the Modeller application. (b) The Ramachandran plot statistics of the modeled three-dimensional structure validated by the PROCHECK program.

TABLE 5: Ramachandran plot statistics of the modeled protein

\begin{tabular}{lc}
\hline Ramachandran plot statistics & $\begin{array}{c}\text { Value } \\
(\%)\end{array}$ \\
\hline Residues in the most favored regions $[A, B, L]$ & 138 \\
$(92.0)$ & $10(6.7)$ \\
Residues in additional allowed regions $[a, b, l, p]$ & $1(0.7)$ \\
Residues in generously allowed regions & $1(0.7)$ \\
{$[\sim a, \sim b, \sim l, \sim p]$} & 150 \\
Residues in disallowed regions & 2 \\
Number of nonglycine and nonproline residues & 8 \\
Number of end residues (excl. Gly and Pro) & 8 \\
Number of glycine residues (shown as triangles) & 168 \\
Number of proline residues & \\
Total number of residues & \\
\hline
\end{tabular}

determines its balance over a broad temperature scale. The GRAVY index determines the proteins' solubility [45]. The negative value of GRAVY (-0.931) indicated the hydrophilic nature of the protein.

3.3. Subcellular Location Determination. The CELLO (v.2.5), PSORTb (v.3.0.2), SOSUI Gram $\mathrm{N}$, and PSLpred tools were utilized for subcellular location assessment of the protein (WP_ 012256288.1). The tools predicted the subcellular location of the protein as a cytoplasmic protein. The HMMTOP (v.2.0) and TMHMM (v.2.0) programs predicted that there were no transmembrane helices in the protein (WP_012256288.1) and emphasized the cytoplasmic location of the protein present in C. aurantiacus (Table 3).

3.4. Functional Annotation of WP_012256288.1. The CDD tool of NCBI characterizes the domain that is found in the identical protein sequences. CD-Search employs RPS-BLAST to assess a test sequence across position-specific rating datasets that have been assembled from conserved domain (CD) alignments contained in the CD protein cluster.
The CD search tool predicted a conserved domain as a translation initiation factor IF-3 (infC, accession no. PRK00028) of the protein WP_012256288.1. IF-3 is one of the crucial elements for the onset of protein synthesis. It attaches to a $30 \mathrm{~S}$ ribosomal subgroup, shifting the balance between $70 \mathrm{~S}$ ribosomes and their 50S and 30S subgroups towards free subunits and thereby increasing the suitability of 30 S subunits where protein synthesis activation starts. Besides, the ScanProsite program predicted a motif (position: 72-85; accession no. PS00938) as IF-3 (gene: infC), which is one of the primary elements required for protein biosynthesis in bacteria [46]. Also, the Pfam program described two different motifs at the positions of 98-181 (Pfam ID: IF3_C; IF-3, C-terminal domain; $e$ value of $2.4 \times 10^{-34}$ ) and 21-90 (Pfam ID: IF3_N; IF-3, N-terminal domain; $e$ value of $\left.4.0 \times 10^{-33}\right)$.

The CDD tool also validated the domains IF3_C and IF3_N at 98-181 and 21-90. The IF3_C (CDD no. pfam00707) is the only member of the superfamily cl29551, whereas the IF3_N (CDD no. pfam05198) is the only member of the superfamily cl04980 as of the conserved protein domain family search feature by the CDD program. The SuperFamily tool predicted the protein WP_012256288.1 (Figure 1) as profoundly associated with the infC superfamily ( $e$ value of $2.09 \times 10^{-98}$ ). The $x$-axis of the diagram displays the location in the amino acid (aa) count protein (beginning at the $\mathrm{N}$-terminus), and the $y$-axis indicates the coiled coil, while the "window" corresponds to the amino acid window which is examined simultaneously (Figure 1).

3.5. Protein-Protein Interaction. The primary focus of protein-protein interactions is acknowledging how cellular systems operate. Such connections allow the filtering, evaluating, and validating of functional genomics data and offering an insightful platform for annotating functional, structural, and evolutionary features of proteins.

The platform can furnish predictions for prospective experiments and map the interactions between different species [47]. The STRING v.11.0 program was performed to determine the protein-protein (pr-pr) interaction. The 


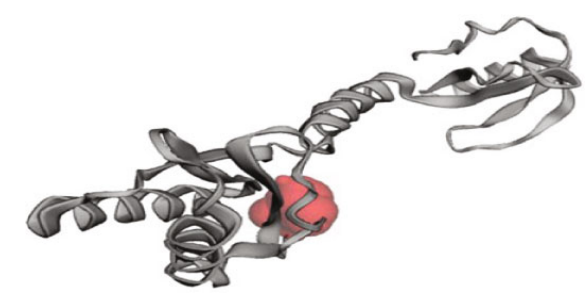

(a)

Chain X

\begin{tabular}{|c|c|}
\hline D R F R I N R I R A R & V R L I D E G T Q V G I P L R E A A MA E E G F D L V V A P N A V P \\
\hline P V C R L L D Y G K F R & 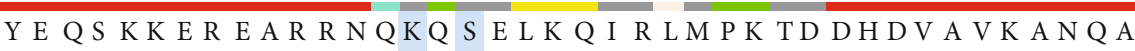 \\
\hline
\end{tabular}

(b)

FIgURe 5: Active site determination: (a) the amino acid residues in the active site (blue color) and (b) active sites of the protein (WP 012256288.1). Also, the "red sphere" indicates the active sites of the protein.

STRING program determined the functional fellows with scores as of rpsM (0.990), rpsE (0.988), rpsK (0.988), rpsS (0.987), rpsI (0.983), rpIT (0.980), rpsC (0.980), rpsJ (0.964), rpsR (0.955), and rpsB (0.951). The rpsM, rpsE, rpsK, rpsS, rpsI, rpIT, rpsC, rpsJ, rpsR, and rpsB are the 30S ribosomal protein S13, 30S ribosomal protein S5, 30S ribosomal protein S11, 30S ribosomal protein S19, ribosomal protein S9 which belongs to the universal ribosomal protein uS9 family, 50S ribosomal protein L20, 30S ribosomal protein S3, 30S ribosomal protein S10, 30S ribosomal protein S18, and ribosomal protein $\mathrm{S}$ which belongs to the universal ribosomal protein uS2 family, respectively (Figure 2).

3.6. Secondary Structure Inquiry. Protein structure and function are strongly connected. The secondary structural components, e.g., helix, coil, sheet, and turn, have an excellent relationship with protein function, structure, and engagement $[48,49]$. The SOPMA program predicted the secondary-structural element of the protein (WP 012256288.1) where the alpha helix (Hh), extended strand $(\mathrm{Ee})$, beta turn $(\mathrm{Tt})$, random coil $(\mathrm{Cc})$ were $121(44.00 \%)$, $45(16.36 \%), 23(8.36 \%)$, and $86(31.27 \%)$, respectively (Table 4). The SPIPRED v.4.0 and DISOPRED v.3.0 tools predicted the sequence plot, secondary structure, and transmembrane topology (Figure 3). The sequence plot from the secondary structure of the IF-3 protein (Figure 3(a)) represents that most of the protein is extracellular, whereas Table 3 reports the protein as cytoplasmic. Further studies are required to unleash the nature of the protein.

3.7. Tertiary-Structure Prediction and Validation. Homology modeling (HM) is a primary method for estimating protein architecture when solely amino acid sequence information is accessible. Protein activities can be derived from the composition of the chain. Using homology modeling (HM) or comparative modeling (CM), scientists would quickly evaluate two closely related sequences' similarities and roles. Sequence similarity to a defined structure is typically repre- sentative of translational and structural similarities to that structure. In the face of these constraints, sequence similarity below 30\% will never provide suitable efficiency in structure prediction [50, 51]. The HHpred is a powerful platform used for distant homology identification and structure estimation, implemented initially as hidden Markov models (HMMs), pioneered by the earliest pairwise comparative analysis of homologous protein profiles. It enables a broad range of repositories, including $\mathrm{PDB}$, Pfam, SCOP, COG, SMART, and CDD. It admits a solitary query array or multiple lineups as input, and it delivers the findings to a PSI-BLAST-like user-friendly interface. Search features are including local or global integration and the detection of secondary systems. HHpred can generate a pair of query prototypes, multiple model alignments with several frameworks from the lookup findings, and 3D structural models from these configurations computed with the Modeller program [52]. The HHpred prognosticated the three-dimensional structure of $\mathrm{WP}_{-}$ 012256288.1 employing the Modeller application (Figure 4). The template (HHpred ID: 5LMN_X) [53] for modeling the three-dimensional structure was chosen based on the most similarity with the IF-3 protein sequence.

The SAVES server's PROCHECK program was utilized for structural quality assessment of the modeled protein, where the arrangement of the $\psi$ angle and the $\varphi$ angle is shown (Table 5, Figure 4). Residues in the most favored regions engulfed 92.0\%, which validated the protein's modeled tertiary structure (WP_012256288.1). Also, residues in additional allowed regions generously allowed regions, disallowed regions, no. of nonglycine and nonproline residues, and no. of end residues (excl. Gly and Pro) were 10 (6.7\%), $1(0.7 \%), 1(0.7 \%), 150$, and 2 , respectively. The no. of glycine residues and the no. of proline residues were similar (8 residues) found in the protein $3 \mathrm{D}$ structure. The C-terminal portion of the protein IF-3 appeared irregular as it contained high charge and repeated regions (Figure 4(a)). Further investigations for describing the functions are required to reveal the mystery, whether due to translation error and/or being a member of the same family. 
3.8. Active Site Determination. The CASTp v.3.0 program predicted 21 different active sites of the modeled protein (Figure 4). CASTp is a database server that can locate areas on proteins, delineate their outline, find the areas' dimensions, and calculate the regions' area. This involves pockets on protein surfaces and vacuums concealed within proteins. The calculation consists of a pocket and volume spectrum or vacuum, both mathematically determined by a solventaccessible surface (surface of Richards) and molecular surface model (surface of Connolly). CASTp could be utilized for the investigation of surface properties and protein operational zones. CASTp provides a pictorial, user-interface versatile, dynamic view and user-submitted constructs on-the-fly measurement [43]. The top active sites of the modeled protein were identified between the area of 85.302 and the volume of 50.667 (Figure 5).

\section{Conclusions}

Comprehending how proteins act is essential for explaining how they operate, and this protein contains IF-3, a crucial factor in protein synthesis considered to initiate protein synthesis. IF- 3 connects to the $30 \mathrm{~S}$ ribosomal subunit and alters the balance between the 70S ribosomes and their 50S and 30S subunits, thereby strengthening the abundance of the $30 \mathrm{~S}$ subunit's affordability of amino acids for the initiation of protein biosynthesis. This investigation reveals the fundamental characteristics including cytoplasmic nature and functional annotation of the protein in association with tertiary structure. Thus, the study findings show the efficiency and scale of further studies on the IF-3 protein of bioinformatics methods used in this investigation.

\section{Abbreviations}

\begin{tabular}{|c|c|}
\hline NCBI: & $\begin{array}{l}\text { National Center for Biotechnology } \\
\text { Information }\end{array}$ \\
\hline PDB: & Protein Data Bank \\
\hline SMS: & Sequence Manipulation Suite \\
\hline CDD: & Conserved Domain Database \\
\hline RPS-BLAST: & Reverse Position-Specific BLAST \\
\hline BLAST: & Basic Local Alignment Search Tool \\
\hline GRAVY: & Grand average of hydropathicity \\
\hline CELLO: & Subcellular localization predictor \\
\hline PSLpred: & $\begin{array}{l}\text { Prediction of subcellular localization of bacte- } \\
\text { rial proteins }\end{array}$ \\
\hline HMMTOP: & $\begin{array}{l}\text { Prediction of transmembrane helices and } \\
\text { topology of proteins }\end{array}$ \\
\hline SPIPRED: & PSI-blast based secondary structure prediction \\
\hline SOPMA: & Self-optimized prediction method \\
\hline CASTp: & $\begin{array}{l}\text { Computed atlas of surface topography of } \\
\text { proteins. }\end{array}$ \\
\hline
\end{tabular}

\section{Data Availability}

The data used to support the findings of this study are available from the submitting or corresponding author on request.

\section{Conflicts of Interest}

The authors have no conflicts of interest to declare.

\section{Authors' Contributions}

A.S.M.S. (Abu Saim Mohammad Saikat) and M.E.U. contributed in conceptualization; M.A.M. contributed in data curation; S.A.A. and T.A. contributed in formal analysis; S.A. contributed in investigation; M.A.M. and S.M. contributed in methodology; S.A.A. and M.A.S.I. contributed in visualization; A.S.M.S. and M.E.U. contributed in writing-original draft; S.M. contributed in writing-review and editing. All authors have read and agreed to the published version of the final manuscript.

\section{Acknowledgments}

This study was equally collaborative among all the authors. Thanks are due to the coauthors who supported proper assistance, financial support, revision, and writing to conduct a successful study. The submission of the manuscript is in ChemRxiv.

\section{References}

[1] B. K. Pierson and R. W. Castenholz, "A phototrophic gliding filamentous bacterium of hot springs, Chloroflexus aurantiacus, gen. and sp. nov.," Archives of Microbiology, vol. 100, no. 1, pp. 5-24, 1974.

[2] J. F. Holden, "Extremophiles: hot environments," in Encyclopedia of Microbiology, pp. 127-146, Elsevier, 2009.

[3] Y. Xin, Y. K. Lu, R. Fromme, P. Fromme, and R. E. Blankenship, "Purification, characterization and crystallization of menaquinol:fumarate oxidoreductase from the green filamentous photosynthetic bacterium Chloroflexus aurantiacus," Biochimica et biophysica acta Reviews on cancer, vol. 1787, no. 2 , pp. 86-96, 2009.

[4] R. G. Feick and R. C. Fuller, "Topography of the photosynthetic apparatus of Chloroflexus aurantiacus," Biochemistry, vol. 23, no. 16, pp. 3693-3700, 1984.

[5] L. He, Y. Wang, L. You, Y. Khin, J. K. H. Tang, and Y. J. Tang, "Glycine cleavage powers photoheterotrophic growth of Chloroflexus aurantiacus in the absence of H2," Frontiers in Microbiology, vol. 6, pp. 1-7, 2015.

[6] J. Oelze and R. C. Fuller, "Temperature dependence of growth and membrane-bound activities of Chloroflexus aurantiacus energy metabolism," Journal of Bacteriology, vol. 155, no. 1, pp. 90-96, 1983.

[7] C. G. Klatt, Z. Liu, M. Ludwig et al., “Temporal metatranscriptomic patterning in phototrophic Chloroflexi inhabiting a microbial mat in a geothermal spring," The ISME Journal, vol. 7, no. 9, pp. 1775-1789, 2013.

[8] K. H. Tang, K. Barry, O. Chertkov et al., "Complete genome sequence of the filamentous anoxygenic phototrophic bacterium Chloroflexus aurantiacus," BMC Genomics, vol. 12, no. 1, pp. 1-21, 2011.

[9] O. A. Zadvornyy, E. S. Boyd, M. C. Posewitz, N. A. Zorin, and J. W. Peters, "Biochemical and structural characterization of enolase from Chloroflexus aurantiacus: evidence for a 
thermophilic origin," Frontiers in Bioengineering and Biotechnology, vol. 3, p. 74, 2015.

[10] B. E. Alber and G. Fuchs, "Propionyl-coenzyme A synthase from Chloroflexus aurantiacus, a key enzyme of the 3hydroxypropionate cycle for autotrophic $\mathrm{CO}_{2}$ fixation*," The Journal of Biological Chemistry, vol. 277, no. 14, pp. 1213712143, 2002.

[11] K. Ratanakhanokchai, J. Kaneko, Y. Kamio, and K. Izaki, "Purification and properties of a maltotetraose- and maltotriose-producing amylase from Chloroflexus aurantiacus," Applied and Environmental Microbiology, vol. 58, no. 8, pp. 2490-2494, 1992.

[12] M. Hügler, C. Menendez, H. Schägger, and G. Fuchs, "Malonyl-coenzyme a reductase from Chloroflexus aurantiacus, a key enzyme of the 3-hydroxypropionate cycle for autotrophic $\mathrm{CO}_{2}$ fixation," Journal of Bacteriology, vol. 184, no. 9, pp. 2404-2410, 2002.

[13] J. Gong, Y. Chen, F. Pu et al., "Understanding membrane protein drug targets in computational perspective," Current Drug Targets, vol. 20, no. 5, pp. 551-564, 2019.

[14] F. Canduri, P. Cardoso Perez, R. Caceres, and W. de Azevedo, "Protein kinases as targets for antiparasitic chemotherapy drugs," Current Drug Targets, vol. 8, no. 3, pp. 389-398, 2007.

[15] A. S. M. Saikat, R. Islam, S. Mahmud et al., "Structural and functional annotation of uncharacterized protein NCGM946K2_146 of Mycobacterium tuberculosis: an in-silico approach," Proceedings., vol. 66, no. 1, p. 13, 2020.

[16] J. H. Pereira, F. Canduri, J. S. De Oliveira et al., "Structural bioinformatics study of EPSP synthase from Mycobacterium tuberculosis," Biochemical and Biophysical Research Communications, vol. 312, no. 3, pp. 608-614, 2003.

[17] C. L. Mills, P. J. Beuning, and M. J. Ondrechen, "Biochemical functional predictions for protein structures of unknown or uncertain function," Computational and Structural Biotechnology Journal, vol. 13, pp. 182-191, 2015.

[18] W. F. de Azevedo Jr., G. C. dos Santos, D. M. dos Santos et al., "Docking and small angle X-ray scattering studies of purine nucleoside phosphorylase," Biochemical and Biophysical Research Communications, vol. 309, no. 4, pp. 923-928, 2003.

[19] W. F. de Azevedo, "Molecular dynamics simulations of protein targets identified in Mycobacterium tuberculosis," Current Medicinal Chemistry, vol. 18, no. 9, pp. 1353-1366, 2011.

[20] W. F. de Azevedo Jr., F. Canduri, V. Fadel, L. G. V. L. Teodoro, V. Hial, and R. A. S. Gomes, "Molecular model for the binary complex of uropepsin and pepstatin," Biochemical and Biophysical Research Communications, vol. 287, no. 1, pp. 277281, 2001.

[21] M. V. Rodnina, "Translation in prokaryotes," Cold Spring Harbor Perspectives in Biology, vol. 10, no. 9, pp. 1-22, 2018.

[22] M. Paci, C. Pon, M. Lammi, and C. Gualerzi, "Structure-function relationship in Escherichia coli translational initiation factors. Characterization of IF-3 by high resolution $1 \mathrm{H}$ NMR spectroscopy.," The Journal of Biological Chemistry, vol. 259, no. 15, pp. 9628-9634, 1984.

[23] D. Brauer and B. Wittmann-Liebold, "The primary structure of the initiation factor IF-3 from Escherichia coli," FEBS Letters, vol. 79, no. 2, pp. 269-275, 1977.

[24] Y. Hua and D. P. Raleigh, "On the global architecture of initiation factor IF3: a comparative study of the linker regions from the Escherichia coli protein and the Bacillus stearothermophilus protein"," Journal of Molecular Biology, vol. 278, no. 4, pp. 871-878, 1998.

[25] C.-S. Yu, C.-J. Lin, and J.-K. Hwang, "Predicting subcellular localization of proteins for Gram-negative bacteria by support vector machines based on n -peptide compositions," Protein Science, vol. 13, no. 5, pp. 1402-1406, 2004.

[26] G. Sanchez, "Science and technology institutions in the learning processes of agri-food production in Argentina," El Sist. Argentino Innovación Inst. Empres and Networks. The Challenge The Creation and Appropriation of Knowledge, vol. 651, pp. 643-651, 2013.

[27] N. Y. Yu, J. R. Wagner, M. R. Laird et al., "PSORTb 3.0: improved protein subcellular localization prediction with refined localization subcategories and predictive capabilities for all prokaryotes," Bioinformatics, vol. 26, no. 13, pp. 1608$1615,2010$.

[28] T. Hirokawa, S. Boon-Chieng, and S. Mitaku, "SOSUI: classification and secondary structure prediction system for membrane proteins," Bioinformatics, vol. 14, no. 4, pp. 378-379, 1998.

[29] M. Bhasin, A. Garg, and G. P. S. Raghava, "PSLpred: prediction of subcellular localization of bacterial proteins," Bioinformatics, vol. 21, no. 10, pp. 2522-2524, 2005.

[30] G. E. Tusnády and I. Simon, "The HMMTOP transmembrane topology prediction server," Bioinformatics, vol. 17, no. 9, pp. 849-850, 2001.

[31] S. Lu, J. Wang, F. Chitsaz et al., "CDD/SPARCLE: the conserved domain database in 2020," Nucleic Acids Research, vol. 48, no. D1, pp. D265-D268, 2020.

[32] M. Kanehisa, S. Goto, S. Kawashima, and A. Nakaya, "The KEGG databases at GenomeNet," Nucleic Acids Research, vol. 30, no. 1, pp. 42-46, 2002.

[33] R. D. Finn, "Pfam: the protein families database," Encyclopedia of Genetics, Genomics, Proteomics and Bioinformatics, vol. 42, pp. D222-D230, 2005.

[34] D. Wilson, M. Madera, C. Vogel, C. Chothia, and J. Gough, "The SUPERFAMILY database in 2007: families and functions," Nucleic Acids Research, vol. 35, no. Database, pp. D308-D313, 2007.

[35] D. Szklarczyk, A. L. Gable, D. Lyon et al., "STRING v11: protein-protein association networks with increased coverage, supporting functional discovery in genome-wide experimental datasets," Nucleic Acids Research, vol. 47, no. D1, pp. D607D613, 2019.

[36] C. Combet, C. Blanchet, C. Geourjon, and G. Deleage, "[email protected]: Network protein sequence analysis," Trends in Biochemical Sciences, vol. 25, no. 3, pp. 147150, 2000.

[37] D. W. A. Buchan and D. T. Jones, "The PSIPRED protein analysis workbench: 20 years on," Nucleic Acids Research, vol. 47, no. W1, pp. W402-W407, 2019.

[38] D. T. Jones and D. Cozzetto, "DISOPRED3: precise disordered region predictions with annotated protein-binding activity," Bioinformatics, vol. 31, no. 6, pp. 857-863, 2015.

[39] B. Webb and A. Sali, "Comparative protein structure modeling using MODELLER," Current Protocols in Protein Science, vol. 86, no. 1, pp. 2.9.1-2.9.37, 2016.

[40] L. Zimmermann, A. Stephens, S. Z. Nam et al., "A completely reimplemented MPI bioinformatics toolkit with a new HHpred server at its core," Journal of Molecular Biology, vol. 430, no. 15, pp. 2237-2243, 2018. 
[41] F. Gabler, S. Z. Nam, S. Till et al., "Protein sequence analysis using the MPI bioinformatics toolkit," Current Protocols in Bioinformatics, vol. 72, no. 1, pp. e108-e130, 2020.

[42] R. A. Laskowski, M. W. Mac Arthur, and J. M. Thornton, "International tables for crystallography," Journal of Applied Crystallography, vol. 16, no. 2, pp. 284-284, 1983.

[43] W. Tian, C. Chen, X. Lei, J. Zhao, and J. Liang, "CASTp 3.0: computed atlas of surface topography of proteins," Nucleic Acids Research, vol. 46, no. W1, pp. W363-W367, 2018.

[44] P. Zhou, "Determining protein half-lives," in Signal Transduction Protocols, pp. 67-77, Springer, 2004.

[45] P. Smialowski, A. J. Martin-Galiano, A. Mikolajka, T. Girschick, T. A. Holak, and D. Frishman, "Protein solubility: sequence based prediction and experimental verification," Bioinformatics, vol. 23, no. 19, pp. 2536-2542, 2007.

[46] D. Liveris, J. J. Schwartz, R. Geertman, and I. Schwartz, "Molecular cloning and sequencing of infC, the gene encoding translation initiation factor IF3, from four enterobacterial species," FEMS Microbiology Letters, vol. 112, no. 2, pp. 211-216, 1993.

[47] A. S. Schwartz, J. Yu, K. R. Gardenour, R. L. Finley Jr, and T. Ideker, "Cost-effective strategies for completing the interactome," Nature Methods, vol. 6, no. 1, pp. 55-61, 2009.

[48] H. B. Uchôa, G. E. Jorge, N. J. Freitas da Silveira, J. C. Camera Jr., F. Canduri, and W. F. de Azevedo Jr., "Parmodel: a web server for automated comparative modeling of proteins," Biochemical and Biophysical Research Communications, vol. 325, no. 4, pp. 1481-1486, 2004.

[49] W. Filgueira de Azevedo, F. Canduri, J. Simões de Oliveira et al., "Molecular model of shikimate kinase from Mycobacterium tuberculosis," Biochemical and Biophysical Research Communications, vol. 295, no. 1, pp. 142-148, 2002.

[50] M. M. Gromiha, R. Nagarajan, and S. Selvaraj, "Protein structural bioinformatics: an overview," Encyclopedia of Bioinformatics and Computational Biology, vol. 1-3, pp. 445-459, 2019.

[51] Z. Xiang, "Advances in homology protein structure modeling," Current Protein \& Peptide Science, vol. 7, no. 3, pp. 217-227, 2006.

[52] J. Söding, A. Biegert, and A. N. Lupas, “The HHpred interactive server for protein homology detection and structure prediction," Nucleic Acids Research, vol. 33, no. Web Server, pp. W244-W248, 2005.

[53] T. Hussain, J. L. Llácer, B. T. Wimberly, J. S. Kieft, and V. Ramakrishnan, "Large-scale movements of IF3 and tRNA during bacterial translation initiation," Cell, vol. 167, no. 1, pp. 133-144.e13, 2016. 